\title{
A Phase I/II Open-Label Multicenter Single-Arm Study of FABLOx (Metronomic 5-Fluorouracil Plus nab-Paclitaxel, Bevacizumab, Leucovorin, and Oxaliplatin) in Patients with Metastatic Pancreatic Cancer
}

Vaibhav Sahai, ${ }^{1, *}$ M. Wasif Saif, ${ }^{2}$ Aparna Kalyan, ${ }^{3}$ Philip A. Philip, ${ }^{4}$ Caio M. Rocha-Lima, ${ }^{5}$ Allyson Ocean, ${ }^{6}$ Michael S. Ondovik, Diane M. Simeone, ${ }^{8}$ Sibabrata Banerjee, Rafia Bhore, ${ }^{7}$ Chrystal U. Louis, and Vincent Picozzi, MD ${ }^{7}$

\begin{abstract}
Purpose: To evaluate safety and preliminary efficacy of metronomic 5-fluorouracil plus nab-paclitaxel, bevacizumab, leucovorin, and oxaliplatin (FABLOx) in patients with newly diagnosed metastatic pancreatic cancer (MPC).

Methods: A total of 12 treatment-naive patients (aged 18-65 years, Eastern Cooperative Oncology Group performance status [ECOG PS] $\leq 1$ ) with MPC received 5 -fluorouracil $180 \mathrm{mg} / \mathrm{m}^{2}$ per day (days $1-14$ continuous infusion); nab-paclitaxel $75 \mathrm{mg} / \mathrm{m}^{2}$, leucovorin $20 \mathrm{mg} / \mathrm{m}^{2}$, and oxaliplatin $40 \mathrm{mg} / \mathrm{m}^{2}$ (days 1, 8, and 15); and bevacizumab $5 \mathrm{mg} / \mathrm{kg}$ (days 1 and 15) administered intravenously in each 28-day cycle. The primary endpoint was incidence of dose-limiting toxicities (DLTs) in cycle 1. Safety was further evaluated as a secondary end-point; preliminary efficacy was also examined.

Results: Two DLTs (grade 3 anemia requiring transfusion and grade 3 mucositis unresponsive to treatment within 4 days of onset) were observed in one of six patients enrolled in dose cohort 1. Cohort 1 was expanded from 6 to 12 patients to further evaluate safety, per the investigators' recommendation. All patients discontinued treatment. The most common grade $\geq 3$ adverse events were abdominal pain, fatigue, mucositis, and decreased neutrophil count. Objective response rate was 33\% (four partial responses). Median progression-free survival (PFS) and overall survival (OS) were 5.6 (95\% confidence interval [Cl], 1.7-11.3) and $9.9(95 \% \mathrm{Cl}, 4.4-13.2)$ months, respectively; 1-year PFS and OS rates were $12.2 \%(95 \% \mathrm{Cl}, 0.7-40.8)$ and $38.9 \%(95 \% \mathrm{Cl}, 12.6-65.0)$.

Conclusion: FABLOx is feasible and tolerable in patients newly diagnosed with MPC. However, preliminary efficacy data are inconclusive for continued investigation in a phase II trial.
\end{abstract}

Keywords: 5-fluorouracil; metastatic pancreatic cancer; combination chemotherapy; nab-paclitaxel

\footnotetext{
${ }^{1}$ Division of Hematology and Oncology, Department of Internal Medicine, University of Michigan, Ann Arbor, Michigan.

${ }^{2}$ Medical Oncology, Northwell Health Cancer Institute, Lake Success, New York.

${ }^{3}$ Department of Medicine, Northwestern University Feinberg School of Medicine, Chicago, Illinois.

${ }^{4}$ Department of Oncology, Karmanos Cancer Institute, Detroit, Michigan.

${ }^{5}$ Department of Oncology, Wake Forest School of Medicine, Bowman Gray Center, Winston-Salem, North Carolina.

${ }^{6}$ Department of Medical Oncology, Weill Cornell Medicine, New York, New York.

${ }^{7}$ Department of Medical Affairs, Celgene Corporation, Summit, New Jersey.

${ }^{8}$ Department of General Surgery, NYU Langone Perlmutter Cancer Center, New York, New York.

${ }^{9}$ Department of Oncology, Virginia Mason Medical Center, Seattle, Washington.

Sahai V, et al. Ann Oncol. 2018;29:suppl_5, mdy151.154. [poster 155].
}

*Address correspondence to: Vaibhav Sahai, MBBS, MS, Division of Hematology and Oncology, Department of Internal Medicine, University of Michigan, 1500 E Medical Center Dr., Ann Arbor, MI 48109, e-mail: vsahai@med.umich.edu

(c) Vaibhav Sahai et al. 2019; Published by Mary Ann Liebert, Inc. This Open Access article is distributed under the terms of the Creative Commons License (http://creativecommons.org/licenses/by/4.0), which permits unrestricted use, distribution, and reproduction in any medium, provided the original work is properly cited. 


\section{Introduction}

Systemic chemotherapy remains the standard of care for patients with metastatic pancreatic cancer (MPC) despite the introduction of novel approaches, such as targeted therapy and immune checkpoint inhibitors, in other malignancies. ${ }^{1,2}$ In 1997, gemcitabine monotherapy was established as the standard of care in MPC, with subsequent trials using combination therapies with a gemcitabine-based backbone failing to demonstrate a clinically and statistically significant improvement in survival. $^{3-8}$ In 2011, the PRODIGE 4/ACCORD 11 trial reported the FOLFIRINOX regimen (leucovorin, 5-fluorouracil [5-FU], irinotecan, and oxaliplatin), and, in 2013, the MPACT trial reported the combination of nab-paclitaxel plus gemcitabine. Each regimen demonstrated significantly longer overall survival (OS) than gemcitabine monotherapy in their respective phase III trials. ${ }^{9-11}$ In PRODIGE 4/ACCORD 11, the median OS was 11.1 months with FOLFIRINOX versus 6.8 months with gemcitabine monotherapy (HR, 0.57 [95\% confidence interval (CI), 0.45-0.73]; $p<0.001) .{ }^{9}$ The MPACT trial demonstrated a median OS of 8.7 months with nab-paclitaxel plus gemcitabine versus 6.6 months with gemcitabine monotherapy (HR, 0.72 [95\% CI, 0.62-0.83]; $p<0.001) .{ }^{11}$ On the basis of these results, either $n a b$-paclitaxel plus gemcitabine or FOLFIRINOX are considered preferred regimens for the first-line treatment of patients with MPC. ${ }^{2}$

Although both regimens demonstrated a survival benefit, the patient's age, performance status and nutritional intake, and the regimen's distinct safety profiles are important considerations when selecting treatments for patients and designing prospective clinical trials. In the PRODIGE 4/ACCORD 11 trial, common grade $3 / 4$ hematological adverse events associated with FOLFIRINOX were neutropenia (45.7\%), thrombocytopenia $(9.1 \%)$, anemia $(7.8 \%)$, and febrile neutropenia (5.4\%); common nonhematological adverse events were fatigue (23.6\%), vomiting (14.5\%), diarrhea (12.7\%), sensory neuropathy $(9.0 \%)$, elevated levels of alanine aminotransferase $(7.3 \%)$, and thromboembolism $(6.6 \%){ }^{9}$ In the MPACT trial, common grade $\geq 3$ hematological adverse events associated with $n a b$-paclitaxel plus gemcitabine were neutropenia (38\%), leukopenia (31\%), thrombocytopenia (13\%), and anemia (13\%); common nonhematological adverse events were fatigue (17\%), peripheral neuropathy $(17 \%)$, and diarrhea $(6 \%){ }^{10,11}$

In an effort to mitigate safety concerns with high-dose intermittent chemotherapy while retaining efficacy of combination regimens, multiple strategies have been used, including metronomic dosing and alternating or in-tandem administration of $n a b$-paclitaxel and FOLFIRINOX. Isacoff et al. evaluated low-dose metronomic chemotherapy with 5-FU, nab-paclitaxel, leucovorin, and oxaliplatin plus bevacizumab in 65 patients with advanced PC and reported the results in a retrospective analysis. ${ }^{12}$ Unfortunately, toxicity was still a concern with this regimen, and 22 patients discontinued treatment due to adverse events; however, efficacy was encouraging, with a median OS of 19 months and an objective response rate of $49 \%$. Similarly, retrospective reports of other metronomic regimens, such as POLF (paclitaxel, oxaliplatin, leucovorin, and 5-FU) and PILF (paclitaxel, irinotecan, leucovorin, and 5-FU) support the continued investigation of low-dose metronomic regimens for the treatment of MPC. ${ }^{13}$

Additional combination therapies have been evaluated in single-arm studies of FOLFOX-A (leucovorin, 5-FU, oxaliplatin, and $n a b$-paclitaxel) $)^{14}$ as well as the alternation or in-tandem administration of nab-paclitaxel and FOLFIRINOX in the GABRINOX, SEENA-1, and NabucCO studies, which all reported safety profiles comparable with those demonstrated in the MPACT and PRODIGE trials. ${ }^{15-17}$ These studies demonstrate that toxicity remains a concern with combination chemotherapy regimens despite promising efficacy.

In this study we report the phase I results of a multicenter single-arm study investigating the regimen of metronomic 5-FU plus nab-paclitaxel, bevacizumab, leucovorin, and oxaliplatin (FABLOx) in patients with newly diagnosed MPC. The oxaliplatin and $n a b$-paclitaxel doses were lower than those administered in the study by Isacoff et al. in an effort to reduce toxicity while maintaining efficacy. ${ }^{18}$ Previous preclinical and clinical investigations have suggested that bevacizumab and other similar antiangiogenic therapies may prolong the antitumor effect of paclitaxel. ${ }^{19,20}$ The primary objective of this study was to determine dose-limiting toxicities (DLTs) of metronomic FABLOx in patients with newly diagnosed MPC.

\section{Methods}

The study was approved by each participating site's institutional review board or independent ethics committee and was conducted in compliance with the International Council for Harmonisation Good Clinical Practice guidelines and with the general ethical principles of the Declaration of Helsinki. Written informed consent was obtained from all patients before study entry. This study was registered on ClinicalTrials.gov under NCT02620800. 


\section{Patients}

Owing to limited experience and the toxicities associated with this five-drug regimen, patient enrollment was limited to those with good performance status, limited comorbidities, and age $\leq 65$ years. Patients (aged 18-65 years) with histologically or cytologically confirmed MPC, an Eastern Cooperative Oncology Group performance status (ECOG PS) of 1 or 0 , and no prior systemic chemotherapy or investigational therapy (other than as a radiosensitizer) for PC were eligible. Adequate blood and organ function as well as measurable disease per Response Evaluation Criteria In Solid Tumors (RECIST) version 1.1 were required for inclusion. ${ }^{21}$ Patients were excluded if they had grade $>1$ peripheral neuropathy, a history of malignancy other than PC in the past 3 years, known brain metastases, or an allergy/hypersensitivity to any investigational products or any of their excipients.

\section{Study design}

In this multicenter single-arm phase I dose de-escalation study, the regimen was given as follows: 5-FU $180 \mathrm{mg} /$ $\mathrm{m}^{2}$ per day on days 1 to 14 through continuous infusion; nab-paclitaxel $75 \mathrm{mg} / \mathrm{m}^{2}$ intravenously (IV) on days 1,8 , and 15; bevacizumab $5 \mathrm{mg} / \mathrm{kg}$ IV on days 1 and 15; leucovorin $20 \mathrm{mg} / \mathrm{m}^{2}$ IV on days 1,8 , and 15 ; and oxaliplatin $40 \mathrm{mg} / \mathrm{m}^{2} \mathrm{IV}$ on days 1,8 , and 15 in each 28 -day treatment cycle (the order of receipt of agents is summarized in Fig. 1; the planned dose de-escalation protocol is summarized in Supplementary Table S1). As noted previously, the oxaliplatin and nab-paclitaxel doses were lower than those administered in the study by Isacoff et al. in an effort to potentially reduce toxicity. ${ }^{18}$ Patients were treated until disease progression, unacceptable toxicity, withdrawal of consent, physician decision, or death.
Assessment of safety end-points

and preliminary efficacy

The primary end-point was the incidence of DLTs, assessed in cycle 1 (DLTs are defined in Supplementary Table S2). A minimum of six patients were to be enrolled in each consecutive dosing cohort. If DLTs occurred in more than or equal to two of six patients, the dose would be de-escalated. Safety was continuously evaluated by incidence and severity of treatmentemergent adverse events (TEAEs) according to the Medical Dictionary for Regulatory Activities and the National Cancer Institute Common Terminology Criteria for Adverse Events version 4.03.

Progression-free survival (PFS) and OS were represented by Kaplan-Meier curves and median survival times with respective 95\% CIs. After treatment discontinuation, each patient was followed up for disease progression and survival every 90 days for a minimum of 18 months. The objective response rate was assessed by computed tomography or magnetic resonance imaging according to RECIST v1.1 criteria; results are presented by frequency and percentages with 95\% CIs reported for the proportion of responsive patients (complete or partial response) from the total number of patients evaluable for response by RECIST criteria.

\section{Statistical methods}

A minimum of six patients could be enrolled in each consecutive dosing cohort, with enrollment of additional patients per the investigators' recommendation based on the totality of the data, to further evaluate any dose level. A total of 12-24 patients were planned for enrollment, depending on the number of dose levels examined and number of patients enrolled at each dose level.

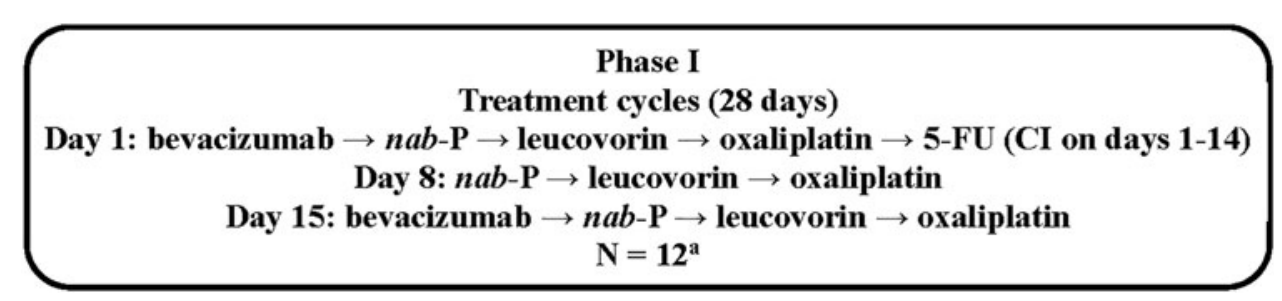

FIG. 1. Study design for phase I. 'anitially, 6 patients were enrolled at dose level 1 , but the cohort was expanded to 12 patients based on investigators' recommendation. 5-FU, 5-fluorouracil; $\mathrm{Cl}$, continuous infusion; DLT, dose-limiting toxicity; nab-P, nab-paclitaxel. 


\section{Results}

Patient disposition

A total of 19 patients were screened for inclusion, but 7 were excluded at screening (MPC not confirmed, unacceptable blood chemistry levels at screening, did not agree to participate in the study, pregnant/nursing [lactating] woman, or other significant medical condition, laboratory abnormality, or psychiatric illness that prevented participation in the study). The treated and response-evaluable populations consisted of 12 patients. All patients discontinued treatment, with the majority discontinuing due to progressive disease $(n=7)$ followed by adverse events $(n=4)$ and withdrawal by patient $(n=1)$.

\section{Baseline characteristics}

Patient demographics and baseline characteristics are described in Table 1 . The median age was 57.5 years and most patients $(75.0 \%)$ were male. The most common sites of metastasis were the liver $(83.3 \%)$, peritoneum (66.7\%), and lung/thoracic cavity (41.7\%).

\section{Safety}

Six patients were enrolled in the first dose cohort. One patient experienced two DLTs (grade 3 anemia requiring a transfusion and grade 3 mucositis unresponsive to medical treatment within 4 days of onset). The starting dose cohort was expanded from 6 to 12 patients to further evaluate the rates of mucositis, per the investigators' recommendation. No additional DLTs were observed in the expanded cohort. Dose de-escalation was not required.

Table 1. Baseline Demographics and Characteristics

\begin{tabular}{lc}
\hline Characteristic & $\boldsymbol{N}=12$ \\
\hline Age, median (range), years & $57.5(41-64)$ \\
Gender, $n$ (\%) & $9(75.0)$ \\
$\quad$ Male & $3(25.0)$ \\
$\quad$ Female & \\
Eastern Cooperative Oncology Group & \\
performance status, $n(\%)$ & $7(58.3)$ \\
0 & $5(41.7)$ \\
1 & \\
Site $(s)$ of metastasis, $n(\%)$ & $10(83.3)$ \\
Liver & $8(66.7)$ \\
Peritoneum & $5(41.7)$ \\
Lungs/mediastinum & $2(16.7)$ \\
Other & \\
No. of metastatic sites & $4(33.3)$ \\
1 & $3(25.0)$ \\
2 & $5(41.7)$ \\
3 & 0 \\
$>3$ & \\
\hline
\end{tabular}

A summary of TEAEs is provided in Table 2. The most common TEAEs of grade $\geq 3$ were abdominal pain, fatigue, mucositis, and decreased neutrophil count. Sepsis was the only grade 4 TEAE (one patient, not suspected to be related to any study drug). One patient experienced a grade 3 serious adverse event of peritoneal infection that worsened to a grade 5 event. Seven patients experienced $\geq 1$ serious TEAE.

\section{Treatment exposure and dose modifications}

All patients were treated at the starting dose level. Treatment exposure and dose modifications for each agent within the FABLOx regimen are summarized in Table 3. The median treatment duration was $27-28$ weeks for all agents in the FABLOx regimen except oxaliplatin, for which the median treatment duration was 22 weeks.

\section{Efficacy}

The objective response rate (best response: complete, or partial response) was $33 \%$ and the disease control rate (complete response, partial response, or stable disease) was $92 \%$; four patients achieved a partial response and seven had stable disease (Table 4). The median PFS was 5.6 months (95\% CI, 1.7-11.3) and the 1-year PFS rate was $12.2 \%$ (95\% CI, 0.7-40.8; Fig. 2). The median OS was 9.9 months (95\% CI, 4.4-13.2) and the 1-year estimated OS rate was 38.9\% (95\% CI, 12.6-65.0\%; Fig. 3).

Table 2. Safety

\begin{tabular}{|c|c|c|}
\hline & & $N=12$ \\
\hline \multirow{2}{*}{\multicolumn{2}{|c|}{$\begin{array}{l}\text { Patients with } \geq 1 \text { grade } \geq 3 \text { TEAE, } n(\%) \\
\text { Patients with } \geq 1 \text { serious TEAE, } n(\%)\end{array}$}} & $12(100.0)$ \\
\hline & & $7(58.3)$ \\
\hline $\mathrm{AE}, n(\%)$ & $\begin{array}{l}\text { Grade } \\
1 / 2^{\mathrm{a}}\end{array}$ & $\begin{array}{c}\text { Grade } \geq 3 \\
\text { (occurring in } \geq 10 \% \\
\text { of patients) }\end{array}$ \\
\hline \multicolumn{3}{|l|}{ Hematological AEs } \\
\hline Neutrophil count decreased & 0 & $3(25.0)$ \\
\hline Anemia & $1(8.3)$ & $2(16.7)$ \\
\hline \multicolumn{3}{|l|}{ Nonhematological AEs } \\
\hline Abdominal pain & $5(41.7)$ & $3(25.0)$ \\
\hline Fatigue & $8(66.7)$ & $3(25.0)$ \\
\hline Mucosal inflammation & $3(25.0)$ & $3(25.0)$ \\
\hline Alanine aminotransferase increased & 0 & $2(16.7)$ \\
\hline Blood alkaline phosphatase increased & $1(8.3)$ & $2(16.7)$ \\
\hline Nausea & $5(41.7)$ & $2(16.7)$ \\
\hline Peripheral sensory neuropathy & $3(25.0)$ & $2(16.7)$ \\
\hline Stomatitis & $4(33.3)$ & $2(16.7)$ \\
\hline
\end{tabular}


Table 3. Treatment Exposure and Dose Modifications

\begin{tabular}{|c|c|c|c|c|c|}
\hline \multirow[b]{2}{*}{ Parameter } & \multicolumn{5}{|c|}{$N=12$} \\
\hline & Bevacizumab & nab-Paclitaxel & Leucovorin & Oxaliplatin & 5-Fluorouracil \\
\hline \multicolumn{6}{|l|}{ Treatment exposure } \\
\hline Treatment duration, median (range), weeks & $27.85(3.7-51.4)$ & $27.00(3.7-51.4)$ & $27.85(3.7-51.4)$ & $22.00(3.7-51.4)$ & $28.00(3.9-53.6)$ \\
\hline Treatment cycles, median (range), $n$ & $7.0(1-13)$ & $7.0(1-13)$ & $7.0(1-13)$ & $5.5(1-13)$ & $7.0(1-13)$ \\
\hline Relative dose intensity, median, \% & 94.02 & 88.25 & 93.64 & 88.41 & 84.35 \\
\hline Cumulative dose, median (range), mg & $\begin{array}{c}4685.50 \\
(500.0-8217.0)\end{array}$ & $\begin{array}{c}1343.63 \\
(222.0-2737.9)\end{array}$ & $\begin{array}{c}413.87 \\
(57.9-728.9)\end{array}$ & $\begin{array}{c}573.73 \\
(115.8-1462.2)\end{array}$ & $\begin{array}{c}14,253.36 \\
(2161.6-25,630.9)\end{array}$ \\
\hline \multicolumn{6}{|l|}{ Dose modifications } \\
\hline Patients with $\geq 1$ dose reduction, $n$ (\%) & 0 & $5(41.7)$ & 0 & $6(50.0)$ & $7(58.3)$ \\
\hline Patients with $\geq 1$ dose delay, $n$ (\%) & $5(41.7)$ & $5(41.7)$ & $5(41.7)$ & $5(41.7)$ & $5(41.7)$ \\
\hline
\end{tabular}

\section{Discussion}

This phase I trial evaluated the safety of the FABLOx regimen in patients with MPC. Results from this study suggest that the FABLOx regimen is both feasible and tolerable in patients with MPC with an ECOG PS of 0 or 1 , good organ function, and no prior systemic chemotherapy. The FABLOx regimen evaluated in this study demonstrated similar tolerability to the combination therapy regimen reported by Isacoff et al. ${ }^{12}$ In the Isacoff et al. study, 22 patients (34\%) discontinued due to toxicity, which is similar to this study, in which four patients (33\%) treated with FABLOx discontinued due to adverse events. ${ }^{12}$

Multidrug cytotoxic regimens have shown improvement in survival in patients with MPC compared with gemcitabine monotherapy but at the expense of increased toxicity. ${ }^{9,10}$ Multiple trials are evaluating alternative cytotoxic regimens in an attempt to further improve efficacy without increasing toxicity. In the single-arm study by Safran et al. at a single institution, the FOLFOX-A regimen resulted in a median survival of 15 months in patients with MPC and had a comparable toxicity profile to that of standard-of-care regimens. ${ }^{14}$ Similarly, in an attempt to reduce toxicity and the early development of chemotherapy resistance,

Table 4. Response Rates

\begin{tabular}{|c|c|}
\hline Outcome, $n$ (\%) & $N=12$ \\
\hline \multicolumn{2}{|l|}{ Best overall response } \\
\hline Complete response & 0 \\
\hline Partial response & $4(33.3)$ \\
\hline Stable disease $\mathrm{a}^{\mathrm{a}}$ & $7(58.3)$ \\
\hline Progressive disease & $1(8.3)$ \\
\hline Objective response rate & $4(33.3)$ \\
\hline $95 \%$ confidence interval & $13.8-60.9$ \\
\hline Disease control rate ${ }^{\mathrm{a}}$ & $11(91.7)$ \\
\hline
\end{tabular}

a few trials have studied alternating gemcitabine- and 5-FU-based regimens. The phase I/II GABRINOX study investigated sequential monthly administration of nab-paclitaxel plus gemcitabine followed by FOLFIRINOX in patients with MPC. The median OS was reported to be 17.8 months, which was encouraging; however, the rates of grade $3 / 4$ thrombocytopenia, neutropenia, and diarrhea were higher than expected. ${ }^{9,10,15}$ In the phase II SEENA-1 study, either nab-paclitaxel plus gemcitabine was followed by modified FOLFIRINOX (no bolus 5-FU), or nab-paclitaxel plus gemcitabine was given alternating with modified FOLFIRI (5-FU, leucovorin, and irinotecan) for up to 48 weeks. ${ }^{17,22}$ The efficacy results were modest (median OS, 12.3 months in all patients and 13.5 months in patients with disease control after 8 weeks of nabpaclitaxel plus gemcitabine), and the safety profile was generally similar to that in the MPACT and PRODIGE 4/ACCORD 11 trials, with common grade $\geq 3$ toxicities, including neutropenia (43\%), fatigue $(22 \%)$, anemia (21\%), and thrombocytopenia (15\%). ${ }^{9,10,17}$ A single-institution extension of the SEENA-1 regimen of alternating nab-paclitaxel plus gemcitabine with FOLFIRI reported improved survival outcomes (median OS, 16.3 months). ${ }^{22}$ Recently, the phase II NabucCO study investigated the effect of modifying FOLFIRINOX by replacing either oxaliplatin (nab-FOLFIRI) or irinotecan (nab-FOLFOX) with nab-paclitaxel. ${ }^{16}$ These modified regimens demonstrated similar survival to that with FOLFIRINOX (median OS, 10.8-13.2 months) but with lower rates of neutropenia.

Overall, these studies examining nab-paclitaxel- and 5-FU-based regimens have demonstrated feasibility and relatively consistent survival outcomes; however, they are limited by their design (single-arm and singleinstitution studies). The survival results with FABLOx 


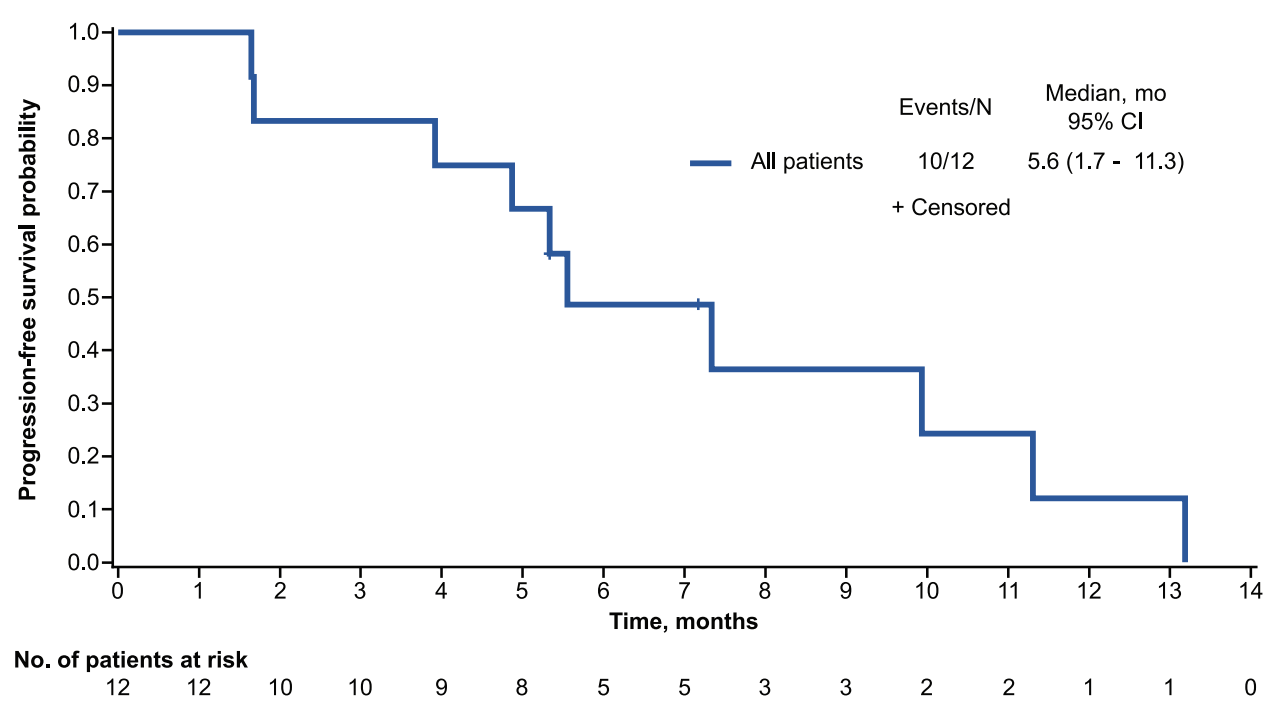

FIG. 2. Progression-free survival.

are similar to those with other prospectively evaluated combination regimens that have reported median OS values ranging from 7.3 to 13.2 months. ${ }^{16,23,24}$ The adverse event profile of FABLOx is also consistent with that reported in previous studies. ${ }^{16,17,25}$ This study supports the relatively consistent outcomes observed with the use of nab-paclitaxel- and 5-FU-based regimens in MPC treatment. Although treatment with FABLOx is feasible in patients with MPC, further investigation is not planned due to the lack of significant improvement in efficacy and the need for exigent 2-week continuous 5-FU infusion. These results further underscore the need for novel agents and greater insight into the biology of PC to understand how to better treat this patient population.

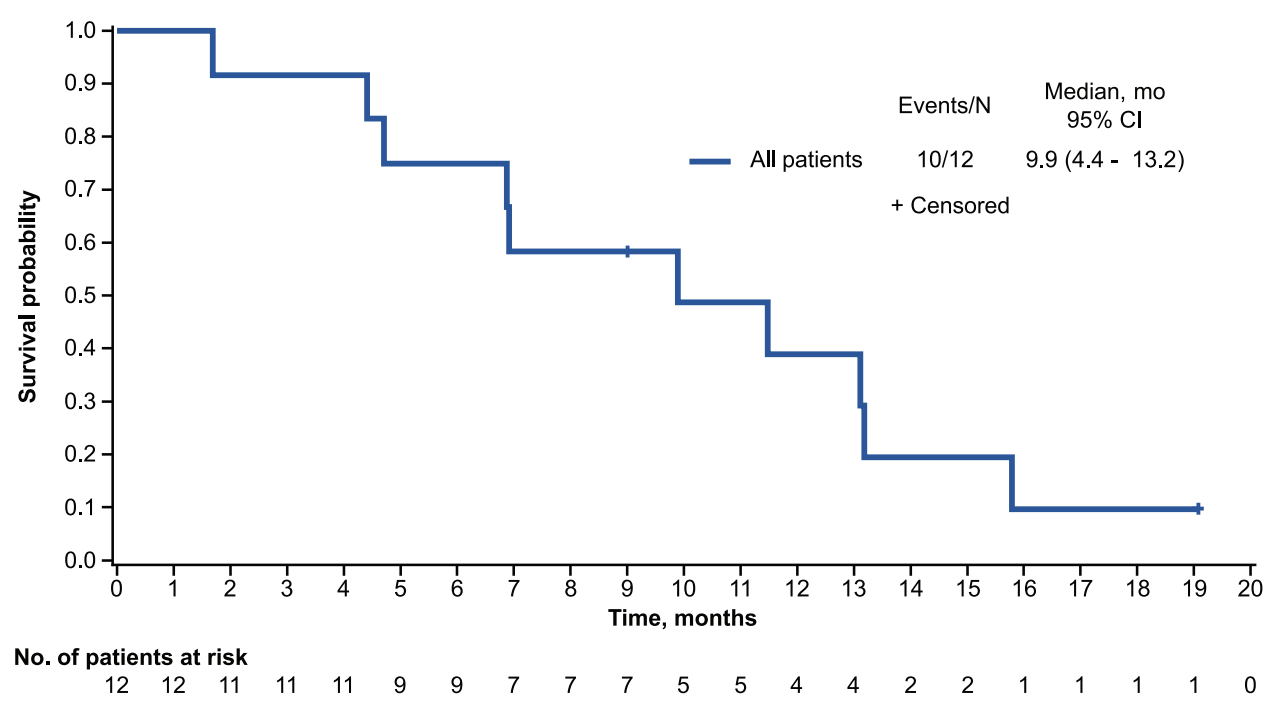

FIG. 3. Overall survival. 


\section{Conclusion}

Results from phase I of this study demonstrate a tolerable safety profile for metronomic FABLOx in patients with newly diagnosed MPC, but preliminary efficacy data are inconclusive for continued investigation in a phase II trial, given the small number of patients in the study.

\section{Acknowledgments}

The authors thank the patients who participated in the study and their families. The study was approved by each participating site's institutional review board or independent ethics committee and was conducted in compliance with the International Council for Harmonisation Good Clinical Practice Guideline and with the general ethical principles of the Declaration of Helsinki. Medical writing assistance was provided by Alessandra Richardson, $\mathrm{PhD}$, of MediTech Media, Ltd, funded by Celgene Corporation. All listed authors were fully responsible for all content and editorial decisions for this article.

\section{Author Disclosure Statement}

V.S. has received institutional research funding from Agios, Celgene, Bristol-Myers Squibb, Ipsen, Incyte, Halozyme, Debiopharm, Rafael, Fibrogen, Medimmune and Clovis, and is/was a consultant for Celgene, Halozyme, Incyte, QED, and NewLink Genetics. M.W.S. is a consultant for EMD Serono; serves on the speakers' bureau for Genentech, Ipsen, Celgene, Novartis, and Sirtex Medical; and has received research funding from Genentech, ImClone Systems, Merrimack, Taiho, Celgene, and Bayer. A.K. has received research funding from Bristol-Myers Squibb and is a consultant for Ipsen, Excelsis, Eisai, and Bristol-Myers Squibb. P.A.P., C.M.R.-L., and D.M.S. have nothing to disclose. A.O. serves on the speakers' bureau for Daiichi Sankyo and is a consultant for Celgene. M.S.O., S.B., R.B., and C.U.L. are employed by Celgene and own stock in the company. V.P. has received grants from AbbVie, Celgene, FibroGen, OncoMed, Aduro, Immunomedics, Lilly, Halozyme, Takeda, and Taiho.

\section{Funding Information}

This work was supported by Celgene Corporation, Summit, New Jersey.

\section{Supplementary Material}

Supplementary Table S1

Supplementary Table S2

\section{References}

1. Ducreux M, Cuhna AS, Caramella $C$, et al. Cancer of the pancreas: ESMO clinical practice guidelines for diagnosis, treatment and follow-up. Ann Oncol. 2015;26(suppl_5):v56-v68.

2. National Comprehensive Cancer Network. NCCN Clinical Practice Guidelines in Oncology. Pancreatic Adenocarcinoma. V2.2019. Available at https://www.nccn.org/professionals/physician_gls/PDF/ pancreatic.pdf Accessed June 18, 2019.

3. Burris HA, 3rd, Moore MJ, Andersen J, et al. Improvements in survival and clinical benefit with gemcitabine as first-line therapy for patients with advanced pancreas cancer: a randomized trial. J Clin Oncol. 1997; 15:2403-2413.

4. Abou-Alfa GK, Letourneau R, Harker G, et al. Randomized phase III study of exatecan and gemcitabine compared with gemcitabine alone in untreated advanced pancreatic cancer. J Clin Oncol. 2006;24: 4441-4447.

5. Colucci G, Labianca R, Di Costanzo F, et al. Randomized phase III trial of gemcitabine plus cisplatin compared with single-agent gemcitabine as first-line treatment of patients with advanced pancreatic cancer: the GIP-1 study. J Clin Oncol. 2010;28: 1645-1651.

6. Cunningham D, Chau I, Stocken DD, et al. Phase III randomized comparison of gemcitabine versus gemcitabine plus capecitabine in patients with advanced pancreatic cancer. J Clin Oncol. 2009;27: 5513-5518.

7. Goncalves A, Viret F, François E, et al. BAYPAN study: a double-blind, phase III randomized trial of gemcitabine plus sorafenib versus gemcitabine plus placebo in patients with advanced pancreatic cancer. J Clin Oncol. 2011;29(15_suppl):4028.

8. Moore MJ, Goldstein D, Hamm J, et al. Erlotinib plus gemcitabine compared with gemcitabine alone in patients with advanced pancreatic cancer: a phase III trial of the National Cancer Institute of Canada Clinical Trials Group. J Clin Oncol. 2007;25: 1960-1966.

9. Conroy $T$, Desseigne $F$, Ychou $M$, et al. FOLFIRINOX versus gemcitabine for metastatic pancreatic cancer. N Engl J Med. 2011;364: 1817-1825.

10. Von Hoff DD, Ervin T, Arena FP, et al. Increased survival in pancreatic cancer with nab-paclitaxel plus gemcitabine. N Engl J Med. 2013;369: 1691-1703.

11. Goldstein D, El-Maraghi RH, Hammel $\mathrm{P}$, et al. Nab-paclitaxel plus gemcitabine for metastatic pancreatic cancer: long-term survival from a phase III trial. J Natl Cancer Inst. 2015;107:pii: dju413 .

12. Isacoff $W H$, Reber HA, Bedford R, et al. Low-dose continuous 5-fluorouracil combined with leucovorin, nab-paclitaxel, oxaliplatin, and bevacizumab for patients with advanced pancreatic cancer: a retrospective analysis. Target Oncol. 2018;13:461-468.

13. Chue BM, La Course BD. Sequential gemcitabine $(G)$, oxaliplatin (O) and irinotecan (I) based weekly metronomic chemotherapy (MC) regimens for the treatment of metastatic pancreatic cancer $(\mathrm{mPC})$ : A community cancer clinic experience. J Clin Oncol. 2019;37(4_suppl): 284.

14. Safran $H$, Charpentier KP, Perez $K$, et al. FOLFOX nab-paclitaxel (FOLFOX-A) for advanced pancreatic cancer. Am J Clin Oncol. 2016;39: 619-622.

15. Assenat E, De La Fouchardiere C, Mollevi C, et al. Sequential treatment with Nab-paclitaxel plus Gemcitabine and Folfirinox in metastatic pancreatic adenocarcinoma: GABRINOX phase II results. J Clin Oncol. 2018;36(15_suppl):4109.

16. Giommoni E, Maiello E, Vaccaro V, et al. Activity and safety of Nab-FOLFIRI and Nab-FOLFOX as first-line treatment for metastatic pancreatic cancer (phase II NabucCO study). J Clin Oncol. 2018; 36(4_suppl):351.

17. Picozzi VJ, Leach JW, Seng JE, et al. Initial gemcitabine/nab-paclitaxel (GA) followed by sequential (S) mFOLFIRINOX or alternating (A) mFOLFIRI in metastatic pancreatic cancer (mPC): The SEENA-1 study. J Clin Oncol. 2017;35(4_suppl):359.

18. Isacoff WH, Reber HA, Hines OJ, et al. Metronomic therapy with 5-FU, weekly nab-paclitaxel, leucovorin, and oxaliplatin, plus bevacizumab for advanced pancreatic cancer: A phase II study. J Clin Oncol. 2012; 30(Suppl 15):e14582. 
19. Miller K, Wang M, Gralow J, et al. Paclitaxel plus bevacizumab versus paclitaxel alone for metastatic breast cancer. N Engl J Med. 2007;357: 2666-2676.

20. Volk LD, Flister MJ, Chihade D, et al. Synergy of nab-paclitaxel and bevacizumab in eradicating large orthotopic breast tumors and preexisting metastases. Neoplasia. 2011;13:327-IN14.

21. Schwartz LH, Litière $S$, de Vries $E$, et al. RECIST 1.1—update and clarification: from the RECIST committee. Eur J Cancer. 2016;62: 132-137.

22. Picozzi VJ, Lin BS, Mandelson MT. Gemcitabine/nab-paclitaxel (G/A) alternating with 5-FU/leucovorin/irinotecan (FOLFIRI) in 1st line metastatic pancreatic cancer (MPC). J Clin Oncol. 2018; 36(15_suppl):e16218.

23. Dahan L, Phelip JM, Le Malicot K, et al. FOLFIRINOX until progression, FOLFIRINOX with maintenance treatment, or sequential treatment with gemcitabine and FOLFIRI.3 for first-line treatment of metastatic pancreatic cancer: A randomized phase II trial (PRODIGE 35PANOPTIMOX). J Clin Oncol. 2018;36(15_suppl):4000.

24. Dubreuil O, Bachet JB, Hammel P, et al. Nab-paclitaxel plus gemcitabine or plus simplified LV5FU2 as first-line therapy in patients with metastatic pancreatic adenocarcinoma: A GERCOR randomized phase II study (AFUGEM). J Clin Oncol. 2017; 35(4_suppl):350.

25. Assenat E, Fouchardiere Cdl, Mollevi C, et al. Gabrinox: A phase I-II of nab-paclitaxel plus gemcitabine followed by folfirinox in metastatic pancreatic adenocarcinoma. Ann Oncol. 2016;27(suppl_6): 207-242.
Cite this article as: Sahai V, Saif MW, Kalyan A, Philip PA, Rocha-Lima CM, Ocean A, Ondovik MS, Simeone DM, Banerjee S, Bhore R, Louis CU, Picozzi V (2019) A phase I/II open-label multicenter single-arm study of FABLOx (metronomic 5-fluorouracil plus nab-paclitaxel, bevacizumab, leucovorin, and oxaliplatin) in patients with metastatic pancreatic cancer, Journal of Pancreatic Cancer 5:1, 35-42,

DOI: 10.1089/pancan.2019.0012.

$\begin{aligned} & \quad \text { Abbreviations Used } \\ & 5-F U= \text {-fluorouracil } \\ & \text { DLTs }=\text { dose-limiting toxicities } \\ & \text { ECOG PS }=\text { Eastern Cooperative Oncology Group performance status } \\ & \text { FABLOx }=\text { metronomic 5-fluorouracil plus nab-paclitaxel, } \\ & \quad \text { bevacizumab, leucovorin, and oxaliplatin } \\ & \text { IV }=\text { intravenously } \\ & \text { MPC }=\text { metastatic pancreatic cancer } \\ & \text { OS }=\text { overall survival } \\ & \text { PFS }=\text { progression-free survival } \\ & \text { PILF }=\text { paclitaxel, irinotecan, leucovorin, and 5-FU } \\ & \text { POLF }=\text { paclitaxel, oxaliplatin, leucovorin, and 5-FU } \\ & \text { RECIST }=\text { Response Evaluation Criteria In Solid Tumors } \\ & \text { TEAE }=\text { treatment-emergent adverse event }\end{aligned}$

\section{Publish in Journal of Pancreatic Cancer}

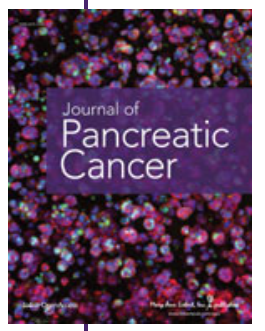

- Immediate, unrestricted online access

- Rigorous peer review

- Compliance with open access mandates

- Authors retain copyright

- Highly indexed

- Targeted email marketing

liebertpub.com/pancan 